\title{
Modelo de realidad virtual de edificios emblemáticos en la Ciudad Universitaria de la UNAH basado en Análisis Espacial con Sistemas de Información Geográfica
}

Eduardo L. Moreno Segura

\section{Resumen}

En la actualidad la Universidad Nacional Autónoma de Honduras se encuentra impulsando fuertemente y con pasos muy significativos el proceso de desarrollo físico en la ciudad Universitaria y sus campus regionales, en tal sentido es necesario el uso de la tecnología para optimizar la planificación, gestión y ordenamiento de su territorio para lo cual la forma de representar sus datos e información viene a tener un alto grado de importancia a la hora de modelar propuestas y tomar decisiones. Una de las representaciones de este tipo de información que en la actualidad ha sido muy efectiva es el modelado fotorrealista 3D que aunado a un sistema de información Geográfica permite modelar futuras afectaciones al contexto y viabilidad de los proyectos entre otros.

El propósito de este proyecto es mostrar mediante modelado $3 \mathrm{~d}$, recorridos de realidad virtual interactivos sobre un Sistema de Información Geográfica, referente al estado de conservación arquitectónico de las principales edificaciones dentro del Campus de la Universidad Nacional Autónoma de Honduras que sirva además para analizar proponer y promover proyectos de: Infraestructura dentro del proceso de plan maestro y desarrollo físico de la UNAH, Ordenamiento Territorial del campus y gestión del mismo.

La metodología utilizada comprende la recopilación de información detallada por cada edificio como ser antecedentes, tipología, planos, fotografías y mediciones entre otras, las cuales fueron procesadas y usadas como insumo para el desarrollo de los modelos 3D georreferenciado incorporado en un SIG, desde el cual se procesarán los análisis de acuerdo a las necedades así como la manipulación en tiempo real y consultas al modelo. 
Los modelos generados como producto en este proyecto, podrán ser usados por planificadores, técnicos, restauradores entre otros, con el fin de hacer estudios, análisis, divulgación de proyectos o futuras predicciones sobre planes de actuación dentro del Campus Universitario.

Palabras clave: Realidad Virtual, Modelo 3d, Sistemas de Información Geográfica, Ordenamiento Territorial.

\section{Abstract}

Today the National Autonomous University of Honduras is promoting heavily and very significant steps the process of physical development in University City and its regional campus, in this sense the use of technology to optimize planning, management and planning is necessary of its territory for which the way of representing data and information comes to have a high degree of importance in shaping proposals and decisions. One of the representations of this type of information today has been very effective is the photorealistic $3 \mathrm{D}$ modeling which together with a geographic information system allows modeling of future damages to context and feasibility of projects among others.

The purpose of this project is to show by $3 \mathrm{~d}$ modeling, interactive virtual reality tours on a Geographic Information System, regarding the state of architectural conservation of the main buildings within the campus of the National Autonomous University of Honduras which also serve to analyze and propose promote projects of Infrastructure in the process of planning master and physical development of the UNAH. The methodology includes the collection of detailed information for each building such as history, typology, plans, photographs and measurements among others, which were processed and used as input for the development of 3D models georeferenced incorporated into the GIS, the which by analysis will be processed according to the needs and the real-time manipulation and queries the model.

The models generated as a product in this project may be used by planners, technicians, restaurateurs and others, in order to make studies, analysand, reporting of projects or predictions about future plans of action within the University Campus.

Keywords: Virtual Reality, 3d model, GIS, Land Management.

Eduardo L. Moreno Segura (eddmorse7@gmail.com),Departamento de Ciencia y Tecnologías de la Información Geográfica,Facultad de Ciencias Espaciales, Universidad Nacional Autónoma de Honduras 


\section{Introducción}

Considerando que la investigación como importante activo capaz de hacer avanzar el desarrollo de metodologías que integran las tecnologías de información y de visualización en 3D aplicadas al ámbito de variedad de disciplinas como son la arquitectura, la historia, la planificación urbana, ordenamiento territorial o simplemente aplicadas al conocimiento de la ciudad, es nuestro punto de partida en este trabajo

Las tecnologías de realidad virtual compatibles con las de un Sistema de Información Geográfica, proporciona a los investigadores la infraestructura fundamental para comenzar a construir ciudades virtuales que pueden recrear un entorno interactivo de simulación y análisis de los lugares urbanos con auténtico realismo, que a la vez pueden integrar todo el conjunto de parámetros medioambientales (físicos sociales y económicos) que requiere la planificación urbana.

En este proyecto "MODELO DE REALIDAD VIRTUAL DE EDIFICIOS EMBLEMÁTICOS EN LA CIUDAD UNIVERSITARIA DE LA UNAH BASADO EN ANÁLISIS ESPACIAL CON SISTEMAS DE INFORMACIÓN GEOGRÁFICA", aplicamos la metodología diseñada tomando como área de estudio el campus Ciudad Universitaria de la UNAH obteniendo modelos tridimensionales y fotorealistas cuyos aportes de información contribuirían a las investigaciones y proyectos desarrollados para el ordenamiento territorial, la gestión, conservación de edificios y desarrollo del centro histórico, así como de verlos proyectados de una forma comprensible e interactiva, dando paso a una buena promoción y divulgación educativa, en diferentes niveles del conocimiento.

\section{Metodología}

La metodología a implementar se basa en un estudio de tipo descriptivo ya que se busca a través de la representación tridimensional de la información geoespacial vinculada a un SIG la descripción y análisis de la infraestructura y crecimiento físico de ciudad universitaria. Es decir que mediante la información descriptiva representada tridimensionalmente y con capacidad de consulta a una base de datos geográfica, se pretende analizar las características particulares como ser el uso e intervención sobre las edificaciones en un periodo de tiempo (2007-2014) y la potencialidad y ventajas que permite este tipo de modelos para los procesos de gestión de patrimonio y desarrollo físico. 
La metodología a seguir comprende 5 grandes aspectos:

- Recopilación y procesado de los datos

- Diseño del Sistema de Información geográfica y modelos 3d de las edificaciones y ambientes físicos construidos dentro de ciudad Universitaria.

- Procesamiento y Análisis de la información utilizando el SIG vinculado a la representación tridimensional de las edificaciones en un espacio de comparación temporal de 7 años (2007-2014).

- Generación de recorridos virtuales con manipulación en tiempo real.

- Divulgación y publicación de resultados.

\section{Variables y su operacionalización:}

Las variables a investigar son: Construcción, Remodelación, Uso, Área y Altura. En el cuadro siguiente se define y operacionalizan las variables.

\begin{tabular}{|l|l|l|}
\hline Variables & Definición Conceptual & $\begin{array}{l}\text { Definición } \\
\text { Operacional }\end{array}$ \\
\hline Construcción & $\begin{array}{l}\text { Se refiere a la fecha exacta en que la edificación fue } \\
\text { construida comprende las dimensiones de Fecha de } \\
\text { Inicio de la edificación y fecha de terminación de la } \\
\text { edificación. }\end{array}$ & $\begin{array}{l}\text { Fecha de } \\
\text { construcción }\end{array}$ \\
\hline Remodelación & $\begin{array}{l}\text { Se refiere a la fecha de restauración arreglo o } \\
\text { modificación de la edificación. }\end{array}$ & $\begin{array}{l}\text { Fecha de } \\
\text { remodelación. }\end{array}$ \\
\hline Uso & $\begin{array}{l}\text { Se refiere al uso que cada edificación tiene y en las } \\
\text { dimensiones de uso original y uso actual }\end{array}$ & $\begin{array}{l}\text { Uso de la } \\
\text { Edificación }\end{array}$ \\
\hline Altura & $\begin{array}{l}\text { Se refiere al área o superficie de construcción de la } \\
\text { edificación }\end{array}$ & $\begin{array}{l}\text { Área de } \\
\text { construcción }\end{array}$ \\
\hline Unidad Académica & $\begin{array}{l}\text { Se refiere a la altura máxima de la edificación tomando } \\
\text { como referencia el nivel de piso terminado +-0.00 }\end{array}$ & Altura del edificio \\
edificación
\end{tabular}

Cuadro 1. Operacionalización y conceptualización de variables. 
Universo, población y muestra:

El Universo de análisis para esta investigación es la Universidad Nacional Autónoma de Honduras. La Población: son los diferentes edificios que comprenden la Ciudad Universitaria. La Muestra, será el campus de ciudad Universitaria.

\section{La Ciudad Universitaria de la Universidad Nacional Autónoma de Honduras (C.U.) es el conjunto de edificios y espacios que conforman el campus principal de la Universidad Nacional Autónoma de Honduras (UNAH), la cual está ubicada en la zona noroeste de la capital, entre el boulevard Suyapa y el Anillo periferico. Se comienza a construir en 1965}

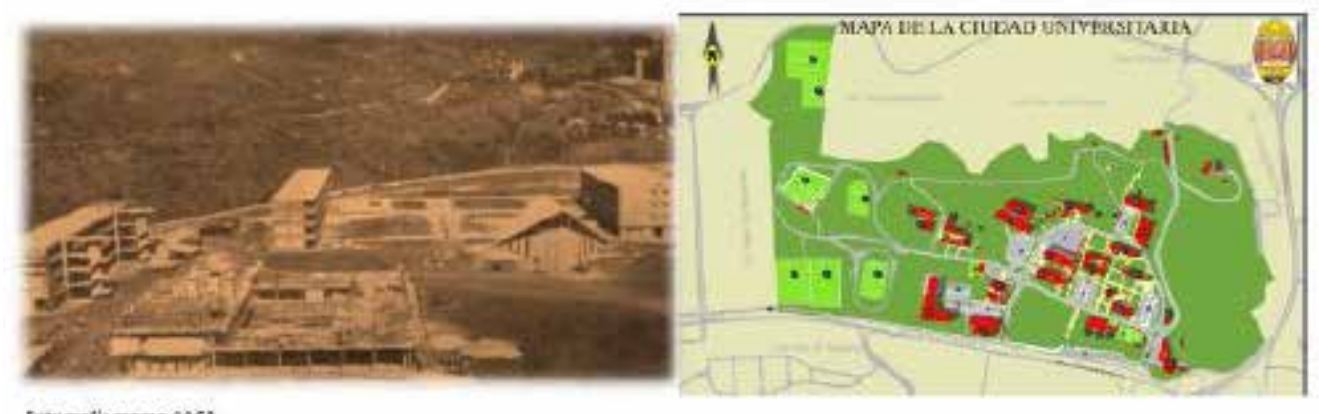

Folugrafierrarzo 1963 .

fuens: hitte:/WWW. sxucraserctvean/showtireas.oho?p=101 190655

\section{Instrumentos:}

Los instrumentos de recolección de la Información serán:

- Cintas métricas para medición de edificaciones en levantamientos directo de campo.

- Receptores GPS para ubicación georreferenciada y altura de edificaciones,

- Cámara Fotográfica digital para levantamiento fotográfico de información.

- Software especializado en SIG, para conformación de la base de datos Geográfica y análisis especial (gvSIG-ArcGIS).

- Software para el modelado 3D (SketchUp, AutoCAD)

- Software Google Heart para la representación tridimensional.

- Software para diseño gráfico y tratamiento digital de imágenes (GIMP), 


\section{Procedimientos y técnicas}

En la investigación comprendió el siguiente procedimiento y técnicas:

\section{Recopilación y procesado de los datos}

- Recolección de datos Geoespaciales y Tabulares

- Se parte de la existencia de la información procesada en el proyecto Modelo de realidad virtual del Centro Histórico del Distrito Central basado en análisis espacial con el SIG para la gestión de centros históricos "parte II", en donde ya se ha logrado la estandarización y clasificación de los datos en parámetros de:

- Identificación: área, zona de estudio (predio de ciudad universitaria-UNAH).

- Calidad de los datos: precisión, fiabilidad.

- Organización de los datos espaciales: vector, raster.

- Referencia espacial: proyección, datum, sistemas de coordenadas.

- Entidad y atributos: información acerca de entidades, atributos, dominio de valores de los atributos.

- Distribución: distribuidor, formatos, precio, otros.

- Referencia de los metadatos: nivel de actualización, institución o persona responsable, otros.

Para la zona de estudio se contempla en primera instancia la selección de las siguientes edificaciones (antigua nomenclatura de edificios de la UNAH):

- Vicerrectoría de Orientación y Asuntos Estudiantiles (VOAE)

- Edificio de Registro

- Auditorio Central

- Edificio de Química y Farmacia

- Edificio 1

- Facultad de Derecho 
- $\quad$ Edificio L1

- Edificio L2

- Facultad de Ciencias Económicas

- $\quad$ Edificio 5

- Edificio 6

- Edificio de Aulas 4B

- Edificio de Aulas $4^{a}$

- Edificio Administrativo

- Facultad de Ciencias Espaciales (FACES)

Recolección de información para el desarrollo de vistas de fachadas:

En esta actividad se seleccionan las edificaciones emblemáticas y de patrimonio Histórico a las cuales se les aplicará la metodología para el desarrollo del modelo. Luego se harán levantamientos directos de campo, como ser medición de fachadas y toma de fotografías de fachadas tratando de evitar lo más posible la perspectiva visual y solamente capturar los detalles en forma ortogonal, en los casos que fuese posible se solicitaran los planos de fachadas de las edificaciones y monumentos que los tengan.

2. Diseño del Sistema de Información geográfica y modelos 3d de las edificaciones y ambientes físicos construidos dentro de ciudad Universitaria.

\section{- Conformación de la base de datos Geográfica}

En esta actividad se diseña conceptualmente la base de datos geográfica declarando los dominios y rangos que permitirán definir las variables seleccionadas así como la configuración del sistema de referencia y cartografía base.

- Levantamiento, ubicación geográfica y fotografías de fachadas de todas las edificaciones seleccionadas en el área de estudio.

En esta actividad se realizará un trabajo de campo donde es necesario ubicar con GPS, fotografiar y medir cada una de las instalaciones dentro del campus Universitario, con el propósito de cotejar y validar los datos que se tienen en gabinete. 
Proceso de edición de imágenes de fachadas (Orto rectificación y limpieza digital de Imágenes).

Luego de tomar las fotos es necesario procesarlas seleccionándolas y transformando la proyección de perspectiva a proyección plana, para luego darles una limpieza digital con un programa de edición de imágenes (por ejemplo Adobe Photoshop o Corel Photo-paint) con el objetivo de eliminar de la fotografía todo elemento que dificulte la visibilidad y apreciación clara de la fachada. Se configuran aspectos como niveles, contraste, brillo y tonalidad de las imágenes.

- Digitalización de fachadas

Se digitalizan de manera precisa todas las fachadas considerando la escala, componentes y detalles arquitectónicos, esto se hace haciendo uso de un programa CAD.

En esta etapa se contará con el documento "Levantamiento e inventario de los edificios de la ciudad universitaria 2008" proporcionado por la Vicerrectoría de Orientación y asuntos estudiantiles en el cual se muestran las características físicas de cada edificio dentro de la UNAH. También se utilizara en los casos que fue necesario, la información vectorial del SIG del Departamento de Ciencia y Tecnologías de la información Geográfica en la Facultad de Ciencias Espaciales.

\section{- Generación de volúmenes en 3D}

En esta actividad se genera el volumen de cada edificación a partir de la planta de techos digitalizada para cada edificio, utilizando el programa Sketchup-Pro en el cual se importará previamente el MDT desde Google Earth lo que permitirá referenciar cada planta de edificios a un sistema de coordenadas real.

- Renderizado de materiales y texturas fotográficas rectificadas.

Una vez generado el volumen del edificio se procederá a importar las texturas de cada fachada desde las fotografías editadas hasta cada una de las caras del modelo ajustando su posición y escala, luego se ocultan las aristas de la geometría del modelo mediante procesos digitales del programa. Esta etapa comprende la incorporación de texturas en edificaciones que han sido remodeladas (texturas pertenecientes a diferentes momentos de su estado físico), lo cual permitirá hacer un análisis comparativo. 
- Completado de la escena.

Comprende la creación y edición de diferentes entornos: desde fondos artificiales con una determinada iluminación, hasta entornos reales construidos en base a modelos de elevación que representan fielmente la orografía del terreno. En este caso se utilizará el entorno tridimensional y foto realista de la plataforma Google Earth.

\section{Análisis de los datos}

Análisis de la información utilizando el SIG vinculado a la representación tridimensional de las edificaciones en un espacio de comparación temporal de 11 años (2002-2013).

- Incorporación de los modelos tridimensionales de edificaciones en el modelo digital del terreno (MDT) en el SIG.

Se exportan los volúmenes $3 \mathrm{~d}$ de las edificaciones al modelo de terreno desarrollado en el SIG para esto se utilizaran puntos $(X, Y)$ de ubicación para cada una de ellas, definidas en un sistema de coordenadas, el cual puede estar asociado a un determinado modelo 3D: Sistema Local, o a un grupo de modelos 3D: Sistema Global, que nos permitirán cualquier manipulación espacial: rotación, escalado y traslación de los mismos.

Para esta etapa se utilizara las potencialidades de la herramienta SIG para generar capas producto del análisis de la información para luego ser representados cartográficamente.

En lo que sigue se ve un estudio comparativo multitemporal donde se ve un despegue significativo en el crecimiento en cuanto a infraestructura a partir del año 2010. 

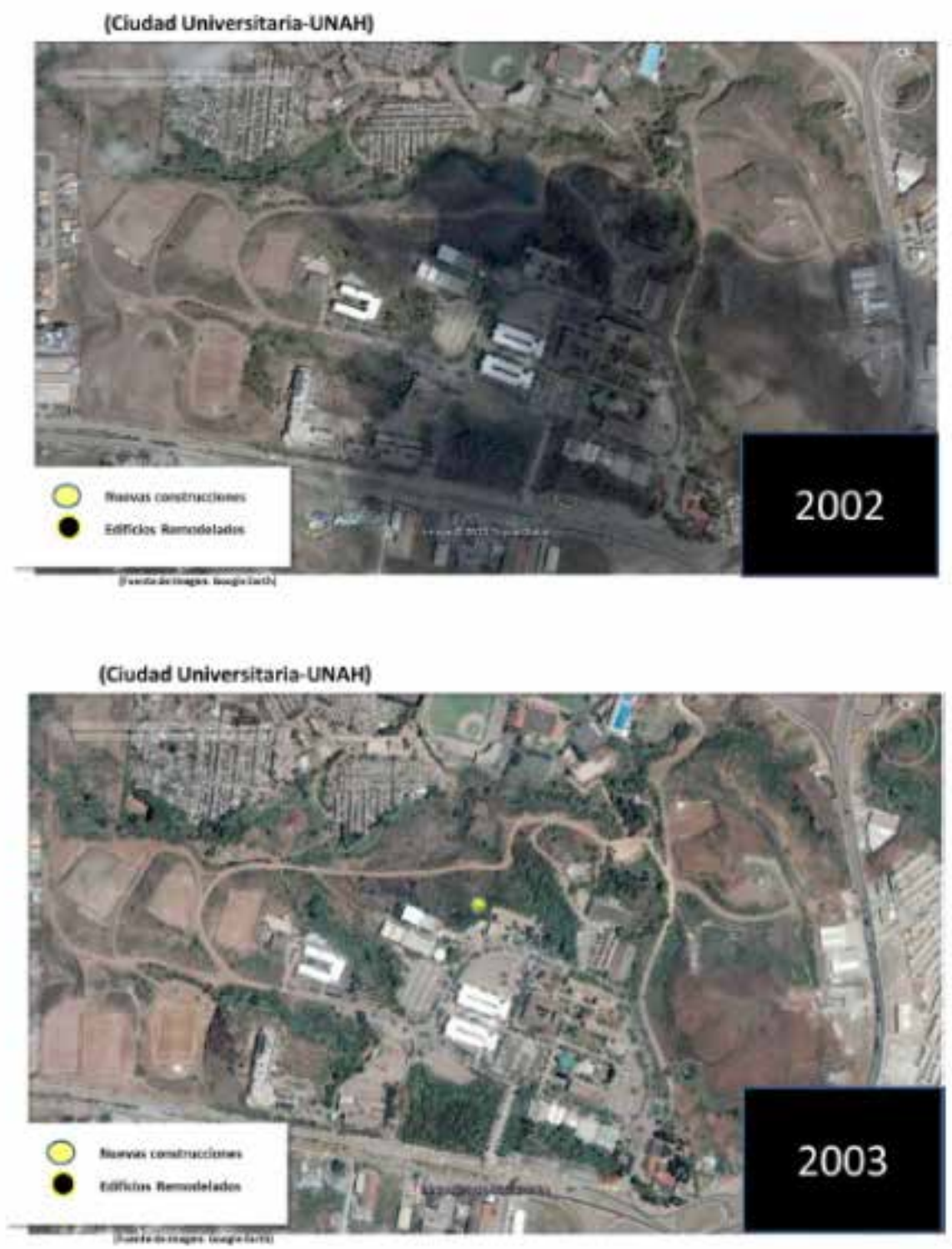


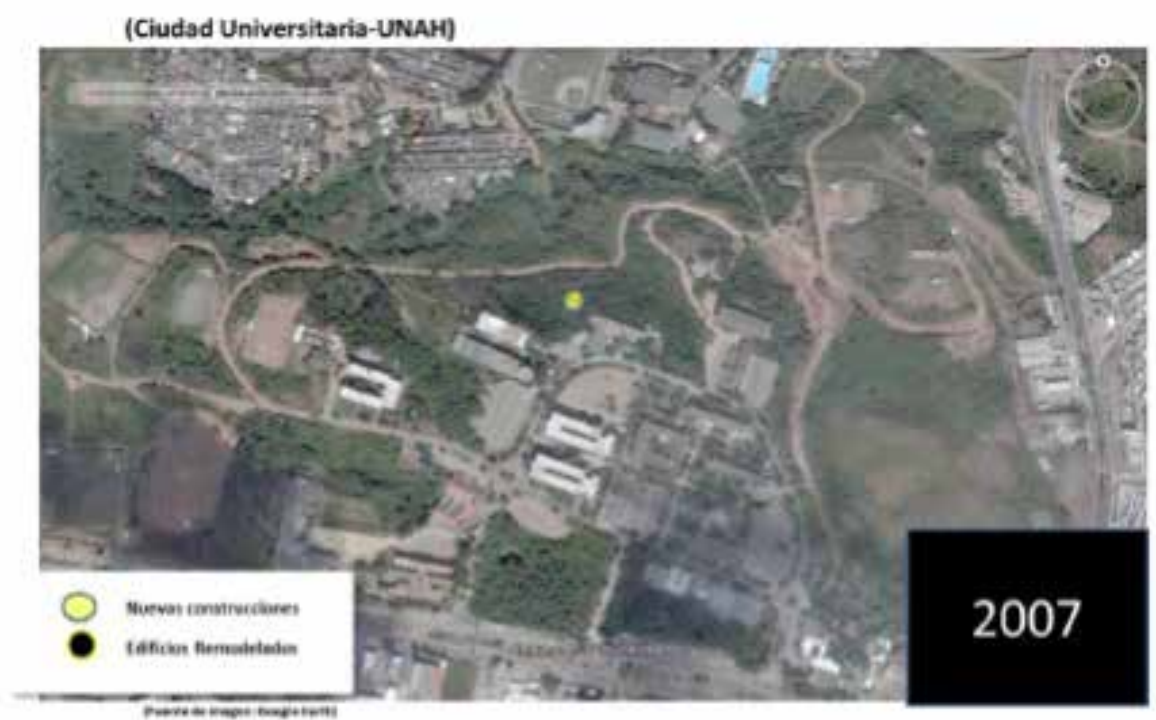

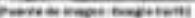

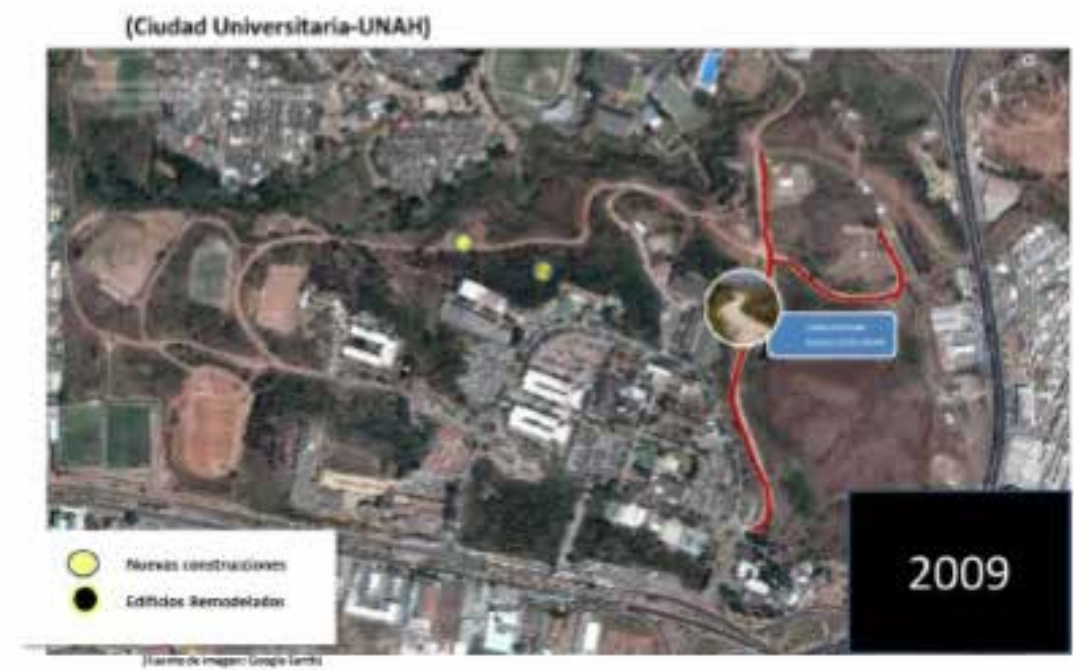



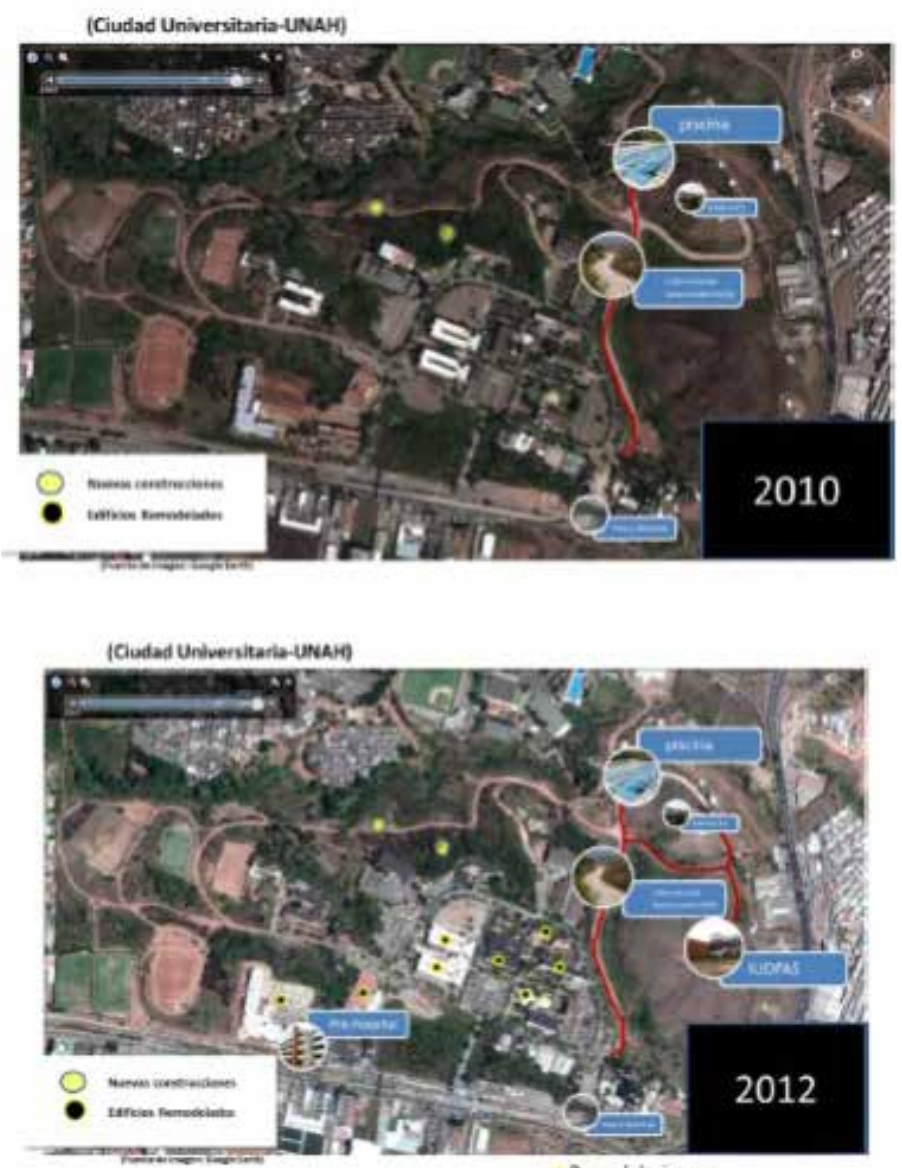

[Ciudad Universitarlo-UNAM)]

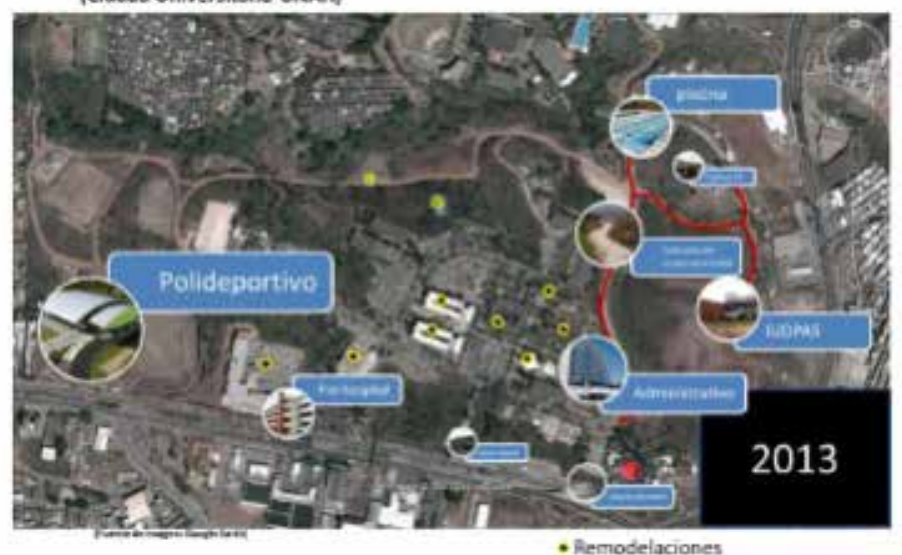


En el siguiente grafico se puede observar que la mayor cantidad de nuevas construcciones y remodelación de edificios antiguos despega significativamente entre los años 2010 y 2013.

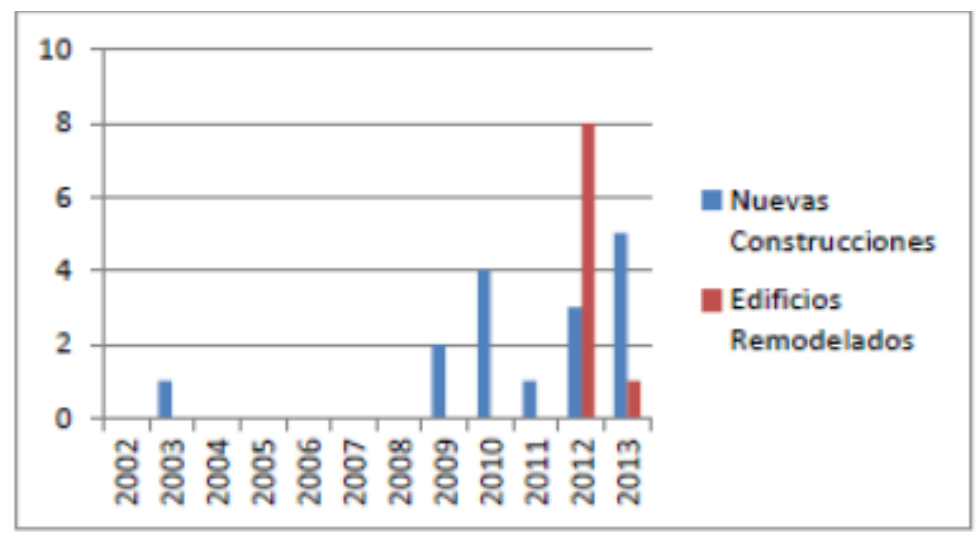

Fotos aéreas de alta resolución no ortorectificadas de ciudad universitaria 20122014.
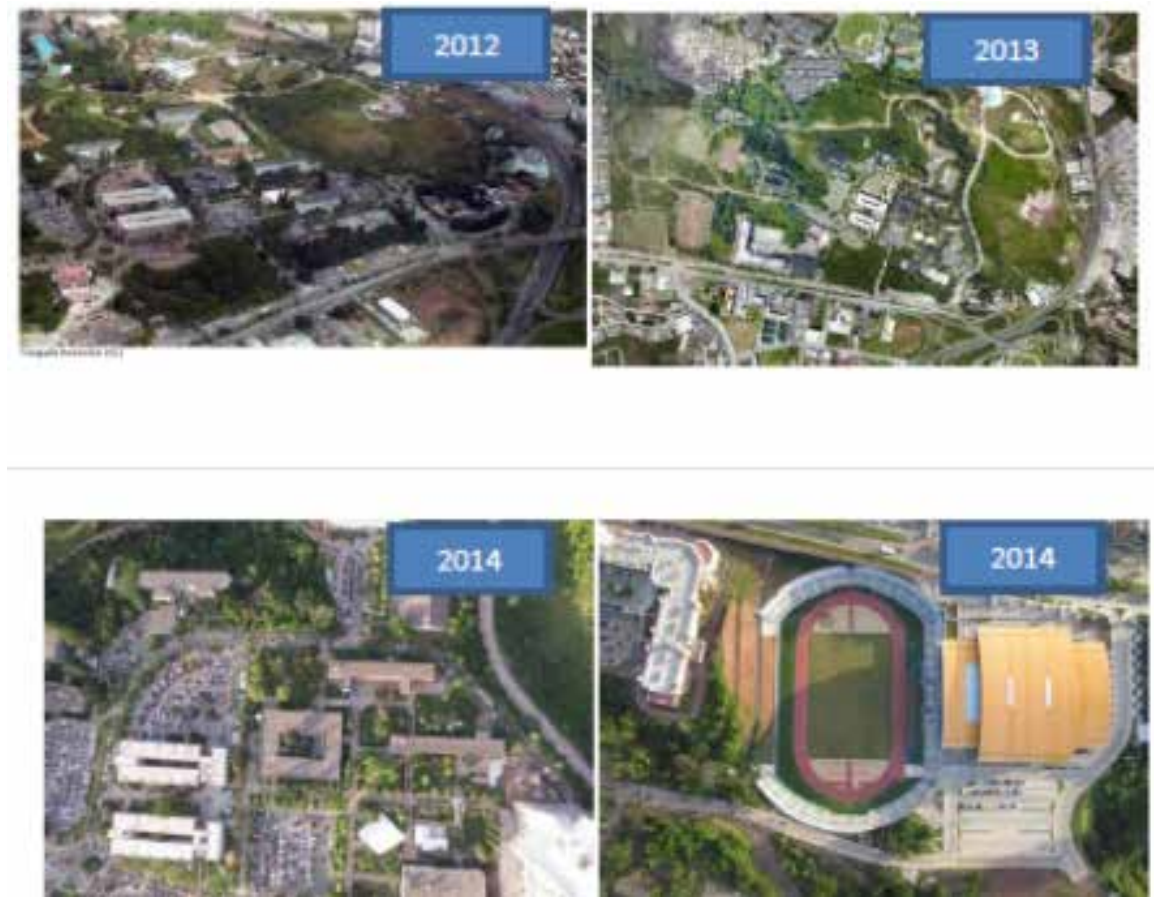
Modelado tridimensional de edificios en ciudad Universitaria

1. VECTORIZACIÓN DE EDIFICACIONES

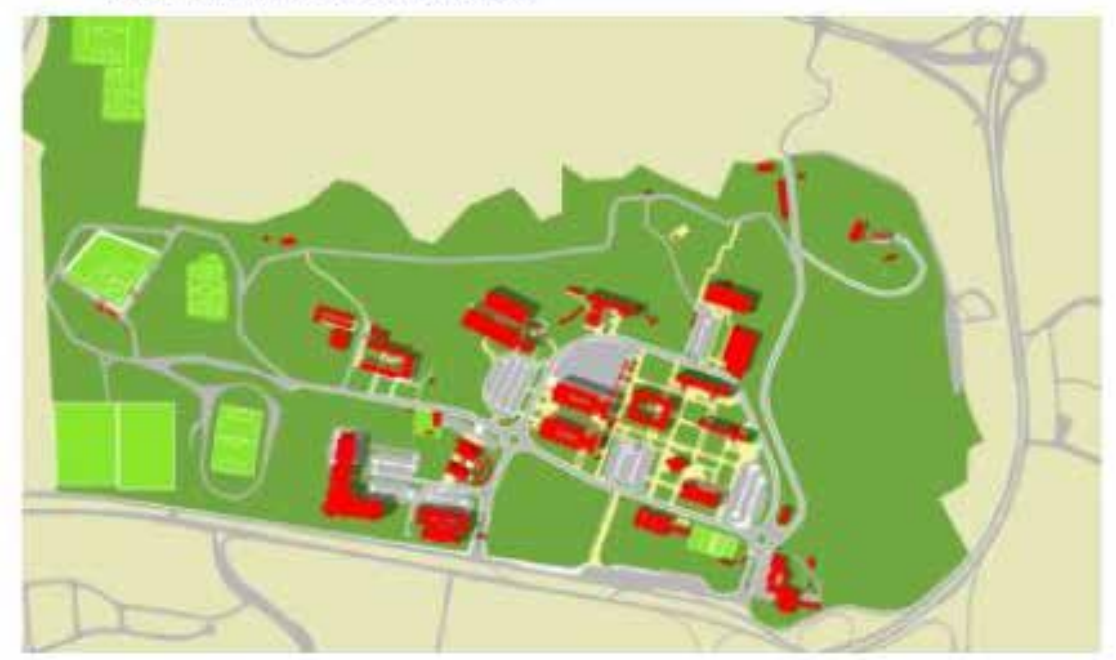

\section{LEVANTAMIENTO, UBICACIÓN GEOGRÁFICA Y FOTOGRAFIAS DE FACHADAS DE LAS} EDIFICACIONES
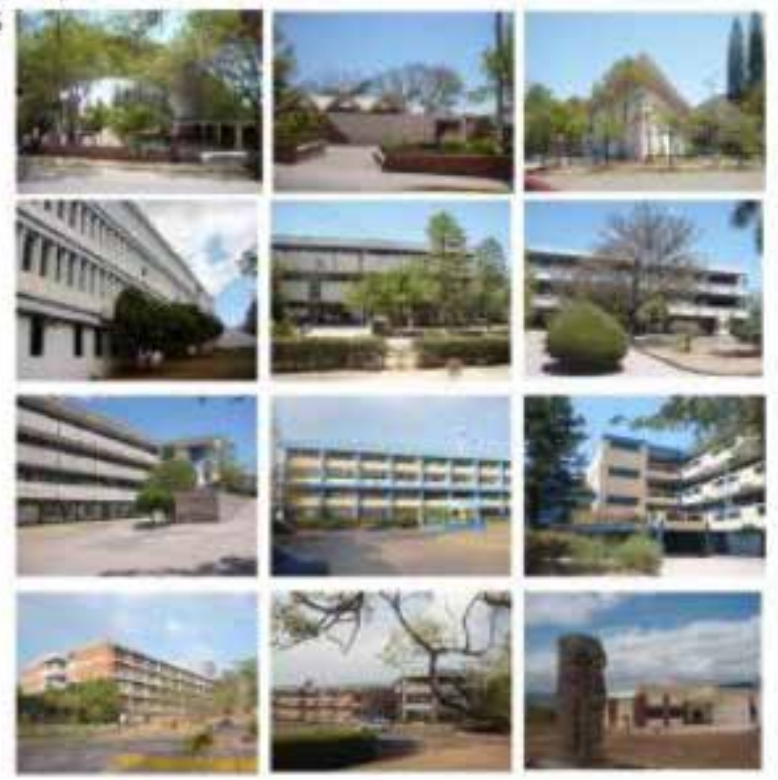


\section{PROCESO DE EDICIÓN DE IMÁGENES DE FACHADAS (ELIMINACIÓN DE PERSPECTIVA Y UMPIEZA DIGITAL DE IMÁGENES).}
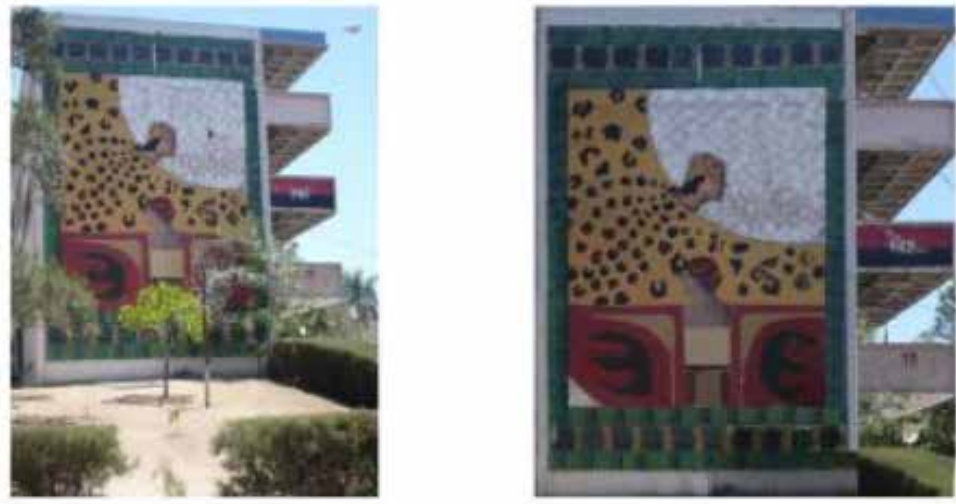

4. Generación de volùmenes en $3 d$

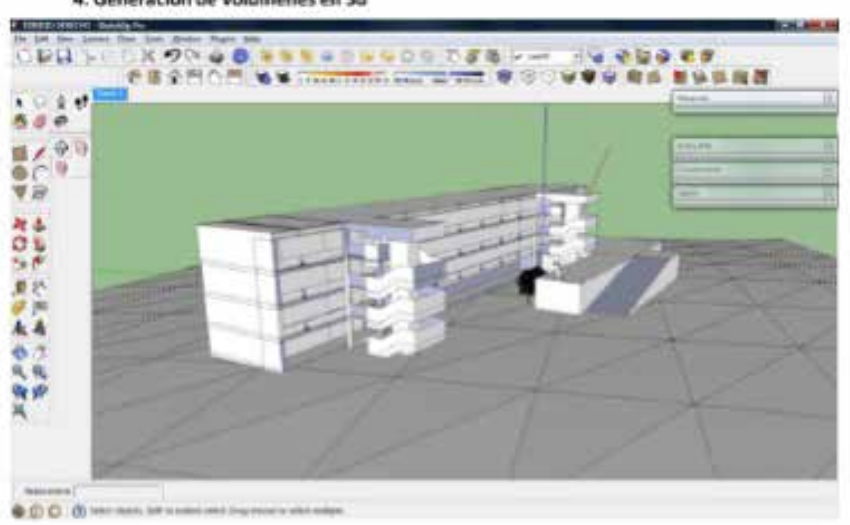

5. Aplicación de materiales y testuras fotogaficas rectificadas.

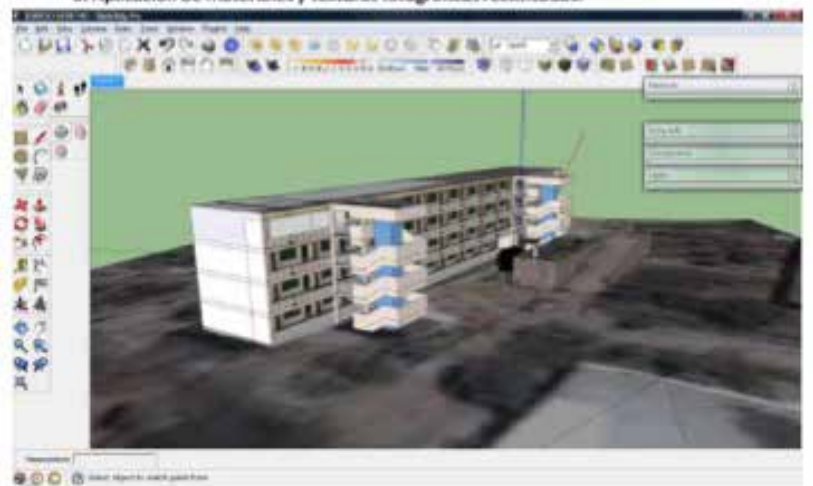


5. Aplicación de materiales y teaturas fotograficas rectificadas.

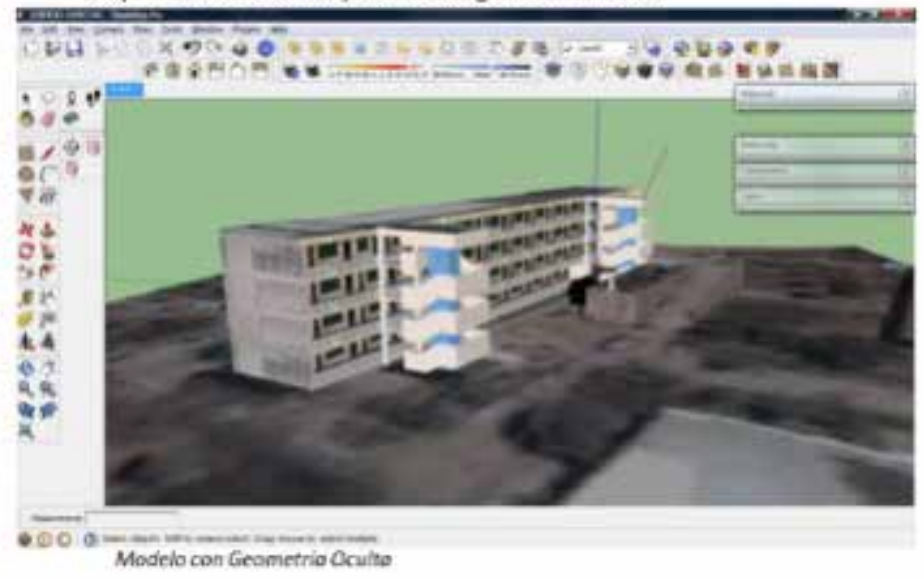

\section{Completado de la escena}

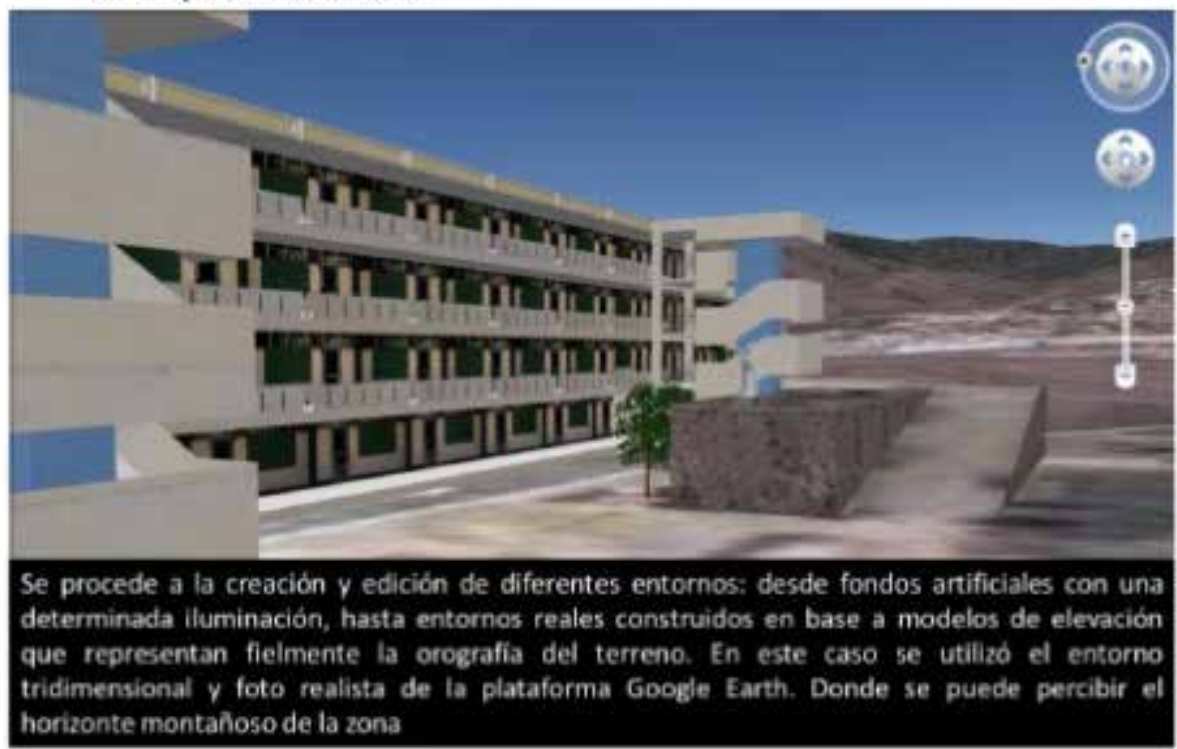




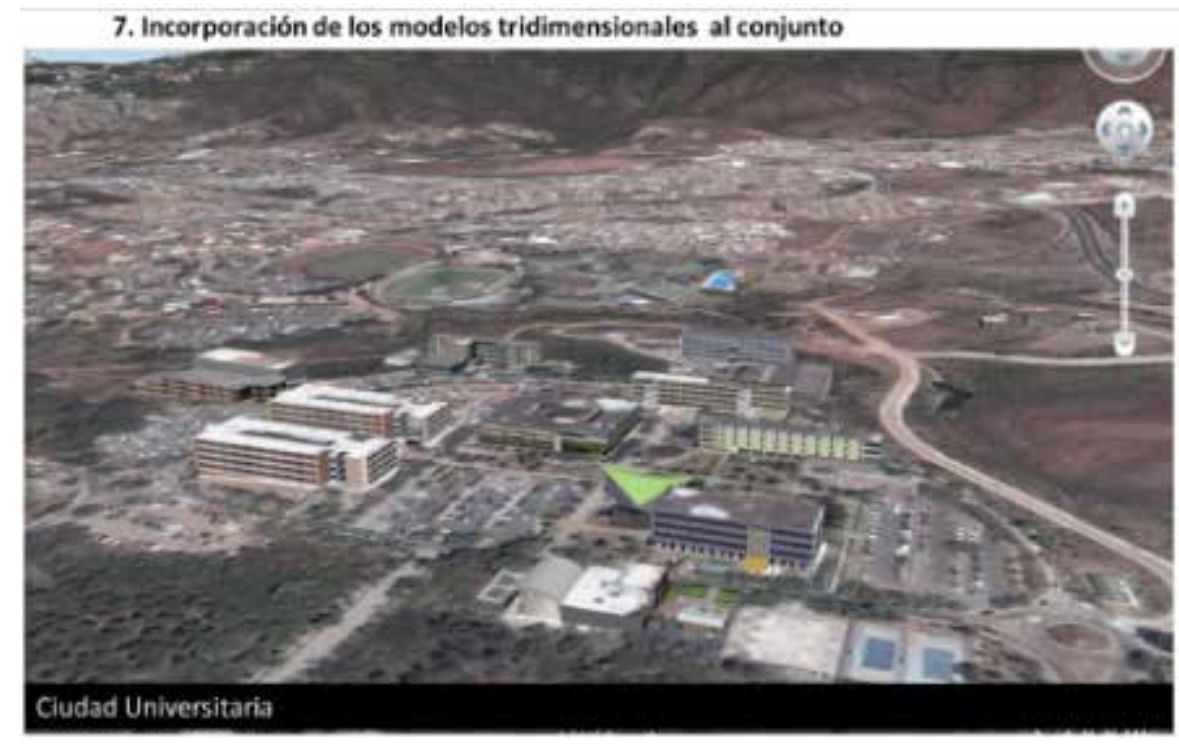

Generación de recorridos virtuales con manipulación en tiempo real.

- Tomas del movimiento y recorridos sobre el modelo.

En esta etapa se procede a definir las rutas del recorrido y ambientación por las diferentes calles proyectadas en el modelo 3D en el SIG para esto se utilizaran opciones especializadas en el programa de SIG que permite grabar el movimiento y definir rutas.

La inmersión en la escena de una cámara virtual y la manipulación de sus diferentes parámetros: focal, eje fotográfico y punto de vista nos permitirá obtener fotografías virtuales desde cualquier posición en la escena 3D. El fotograma virtual resultante podrá ser almacenado y utilizado en posteriores análisis de perspectiva. 
8. Tomas del movimiento y recorridos sobre el modelo.
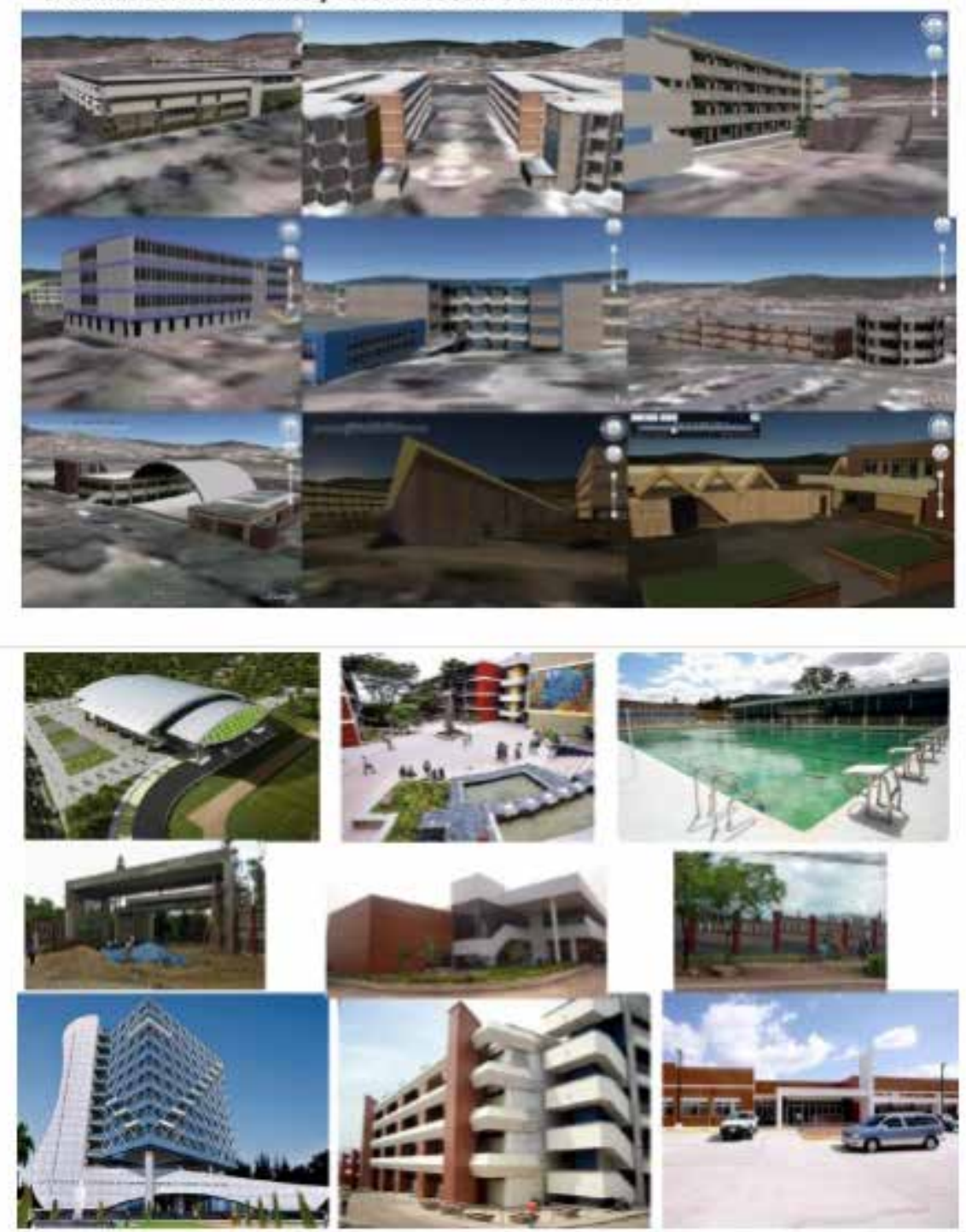

\section{Conclusiones}

La historia arquitectónica de las edificaciones dentro del Centro Histórico de Tegucigalpa y la Ciudad Universitaria de la UNAH puede ser mejor ilustrada a través de estos modelos $3 \mathrm{~d}$ y mejor descrita y analizada con su incorporación a los sistemas de información Geográfica. 
El modelo tridimensional georreferenciado de las instalaciones de la UNAH representa una herramienta muy útil en el proceso de desarrollo físico de la misma ya que en él se puede proyectar y analizar infraestructura nueva y remodelaciones que se pretendan desarrollar a futuro.

La visita Virtual 3D de las áreas de estudio que este proyecto ha generado, promoverá la socialización y conocimiento de las edificaciones dentro de cada una, con múltiples propósitos en el Ordenamiento Territorial, con miras entre otras cosas a concienciar la población sobre el patrimonio tangible con que cuenta.

\section{Bibliografia}

- ALEGSA. (2010).Diccionario de Informática. Recuperado el 16 de octubre de 2013, de http://www.alegsa.com.ar/Dic/modelo\%20en\%203d.php

- Barrera, G. (2009). Realidad Virtual. Recuperado el 15 de octubre de 2013, de http://giselle9229.blogia.com/2009/102701-...realidad-virtual....php.

- BOSQUE SENDRA, J. (1992) Sistemas de Información Geográfica. Madrid. Rialp.

- Consejo de Europa, Conferencia Europea de Ministros responsables de Ordenación del Territorio (CEMAT).(1983). Carta Europea de Ordenación del Territorio.Torremolinos, España.

- Goodchild, M.F., and K.K. Kemp, eds. (1990). NCGIA Core Curriculum in GIS. National Center for Geographic Information and Analysis, University of California, Santa Barbara CA.

- S. Castromonte, J.,(2010). Realidad Virtual. Recuperado el 3 de octubre de 2013, de http://style4ce.blogspot.com/ 